\title{
X-ray diffraction from flight muscle with a headless myosin mutation: implications for interpreting reflection patterns
}

\author{
Hiroyuki Iwamoto $^{1 *}$, Károly Trombitás ${ }^{2}$, Naoto Yagi ${ }^{1}$, Jennifer A. Suggs ${ }^{3}$ and Sanford I. Bernstein ${ }^{3}$ \\ 'Research and Utilization Division, Japan Synchrotron Radiation Research Institute, SPring-8, Hyogo, Japan \\ 2 Veterinary and Comparative Anatomy, Pharmacology and Physiology, Washington State University, Pullman, WA, USA \\ ${ }^{3}$ Department of Biology, Molecular Biology Institute, Heart Institute, San Diego State University, San Diego, CA, USA
}

\section{Edited by:}

Julien Ochala, King's College

London, UK

\section{Reviewed by:}

Han-Zhong Feng, Wayne State

University School of Medicine, USA

Thomas Charles Irving, Illinois

Institute of Technology, USA

\section{*Correspondence:}

Hiroyuki Iwamoto, Research and

Utilization Division, Japan

Synchrotron Radiation Research

Institute, SPring-8, 1-1-1 Kouto,

Sayo-cho, Sayo-gun, Hyogo

679-5198, Japan

e-mail: iwamoto@spring8.or.jp
Fruit fly (Drosophila melanogaster) is one of the most useful animal models to study the causes and effects of hereditary diseases because of its rich genetic resources. It is especially suitable for studying myopathies caused by myosin mutations, because specific mutations can be induced to the flight muscle-specific myosin isoform, while leaving other isoforms intact. Here we describe an X-ray-diffraction-based method to evaluate the structural effects of mutations in contractile proteins in Drosophila indirect flight muscle. Specifically, we describe the effect of the headless myosin mutation, $\mathrm{MhC}^{10}-$ Y97, in which the motor domain of the myosin head is deleted, on the $X$-ray diffraction pattern. The loss of general integrity of the filament lattice is evident from the pattern. A striking observation, however, is the prominent meridional reflection at $d=14.5 \mathrm{~nm}$, a hallmark for the regularity of the myosin-containing thick filament. This reflection has long been considered to arise mainly from the myosin head, but taking the 6th actin layer line reflection as an internal control, the 14.5-nm reflection is even stronger than that of wild-type muscle. We confirmed these results via electron microscopy, wherein image analysis revealed structures with a similar periodicity. These observations have major implications on the interpretation of myosin-based reflections.

Keywords: myosin mutation, insect flight muscle, synchrotron radiation, X-ray diffraction, Drosophila, electron microscopy

\section{INTRODUCTION}

Despite the long phylogenic distance between humans and insects, striking similarities exist in the structure of their muscles. Both the skeletal or cardiac muscles of vertebrates and somatic muscles of insects are cross-striated, have similar sarcomeric structure and are regulated by the thin-filament-based system involving troponin and tropomyosin. In this respect, Drosophila melanogaster is one of the best-suited model animals to study congenital myopathies that occur in humans, owing to its rich genetic resources and ease of breeding. Its indirect flight muscle is the preferred target, because it is the bulkiest of all muscles in the insect, and many of its constituent contractile proteins, including myosin and actin, are expressed as tissue-specific isoforms (Bernstein et al., 1993). Therefore, the mutations or knock-outs of these isoforms are usually viable because non-flight muscle isoforms remain intact.

Here we evaluate the structural consequences of the headless mutant of flight muscle myosin isoform, in which an adult myosin rod transgene (Y97) is expressed in $M h c^{10}$ myosin-null background (Cripps et al., 1999). All of the motor domain and a part of the binding site for the essential light chain are missing from the rod molecule, but the binding site for the regulatory light chain remains intact (Cripps et al., 1999). Electron microscopy of the flight muscle in this mutant has shown that the rod molecules form thick filaments with hollow centers as in wild type, and that these thick filaments are often surrounded by thin filaments and form a hexagonal lattice, but the integrity of the whole myofibrils is inferior to that in wild type (Cripps et al., 1999).

We adopt another approach to evaluate the structure of the mutated flight muscle, i.e., X-ray diffraction. The technique of $\mathrm{X}$-ray diffraction is especially suitable for reporting the regularity of arrangement of contractile proteins in myofilaments, and also the spatial arrangement of myofilaments within a sarcomere. The basic knowledge about X-ray diffraction from muscle was initially established by using bulky isolated whole muscles from frog (e.g., Huxley and Brown, 1967), but brighter X-ray sources, including synchrotron radiation facilities, and more advanced sensitive detectors have made it possible to record X-ray diffraction patterns from wider varieties of muscle specimens with much shorter exposure times. Compared with other techniques, X-ray diffraction can be applied to samples under physiological conditions, and time-resolved measurements are possible (for more detailed explanations for the synchrotron-based X-ray diffraction technique and comparisons with other techniques, refer to Oiwa et al., 2009). In fact, the X-ray diffraction technique has been applied to biopsied specimens of human muscle with congenital myopathies (Ochala et al., 2010; Ochala and Iwamoto, 2013), specimens from transgenic mice with expressed mutations found in human myopathy patients (Ochala et al., 2011, 2013, 2014; Lindqvist et al., 2012, 2013) and rat disease models 
(Corpeno et al., 2014). The intense and well-oriented X-rays from the third-generation synchrotron radiation facilities have made it possible to record full 2-D diffraction patterns of Drosophila indirect flight muscles (Irving and Maughan, 2000; Dickinson et al., 2005; Iwamoto et al., 2007).

Here we tested several newly developed techniques to record X-ray diffraction patterns from glycerinated Drosophila flight muscle specimens, which are too small for ordinary mounting techniques for longer muscle fibers, and the qualities of the obtained diffraction patterns were compared. We applied these techniques combined with electron microscopy analysis to the $M h c^{10}-Y 97$ mutant. This mutant is especially suited for studying the role of the myosin motor domain on the general architecture of sarcomeres, and its contributions to the intensities of the myosin-based reflections. The unexpected findings presented here lead to important implications for the interpretation of muscle X-ray diffraction in general.

\section{MATERIALS AND METHODS SPECIMEN}

The strains $M h c^{10}$ (homozygous myosin null in the indirect flight muscles; Collier et al., 1990) and $M h c^{10}-Y 97$ (homozygous myosin null in the indirect flight muscles and homozygous for a transgene expressing headless myosin specifically in these muscles; Cripps et al., 1999) of D. melanogaster were verified as to myosin protein content and maintained at San Diego State University. Flies were transferred to SPring-8, and were maintained there for X-ray diffraction studies. The wild type of D. melanogaster (Hikone-R strain) was obtained from Ehime University (Iwamoto et al., 2007). Insects other than Drosophila were collected at the campus of SPring-8.

For X-ray diffraction studies, the whole thoraces of Drosophila adults were glycerinated in a 50\% mixture of glycerol and a relaxing solution (for composition see Iwamoto, 2009; Iwamoto et al., 2010) containing phenylmethylsulfoxide and protease inhibitor cocktail (P8340, Sigma-Aldrich, St. Louis, USA), and stored at $20^{\circ} \mathrm{C}$ in a freezer. The flight muscles of other insects were treated in a similar way. The methods of mounting of Drosophila specimens for X-ray recording are detailed in the Results section. Flight muscle fibers of larger insects were mounted as described (Iwamoto et al., 2001, 2010; Iwamoto, 2009).

\section{X-RAY DIFFRACTION RECORDINGS}

$\mathrm{X}$-ray diffraction patterns of flight muscle were recorded the BL45XU small-angle scattering beamline of SPring-8 (Fujisawa et al., 2000). Details of the methods of recording have been described (Iwamoto et al., 2001, 2003; Iwamoto, 2009). Briefly, the specimens were placed in a rigor solution with a composition described previously (Iwamoto, 2000), except for samples from horsefly, which were placed in a relaxing solution (Iwamoto, 2009). In addition, the solution contained $5 \mathrm{mM}$ dithiothreitol to reduce radiation damage. Monochromatized X-ray beams (wavelength, 0.09 or $0.1 \mathrm{~nm}$; flux, $10^{12}$ photons/s, beam size, $0.3 \times 0.2 \mathrm{~mm}$ ) were irradiated to the specimen, and the patterns were recorded by using a cooled CCD camera (C488050, Hamamatsu Photonics, Hamamatsu, Japan) in combination with an image intensifier (VP5445MOD, Hamamatsu Photonics).
The time for single exposure was $1 \mathrm{~s}$. A maximum of 20 exposures were recorded from a single specimen, and the patterns were summed. The background scattering was subtracted by the method described in Iwamoto et al. (2003, 2010).

\section{ELECTRON MICROSCOPY}

Indirect flight muscle bundles for electron microscopic observations were dissected at San Diego State University from 2-day-old flies in relaxing solution (Peckham et al., 1990) containing 1\% Triton X-100 on ice. After $3 \mathrm{~h}$, the liquid was replaced successively by two washes of fresh relaxing solution containing 50\% glycerol and a dissolved Roche protease inhibitor cocktail tablet (aprotinin, leupeptin, EDTA, pefablock). Muscles were stored at $-20^{\circ} \mathrm{C}$ and shipped to Washington State University on ice, where they were fixed with 3\% glutaraldehyde, then postfixed with $1 \%$ osmium tetroxide in $100 \mathrm{mM}$ phosphate buffer, $10 \mathrm{mM} \mathrm{MgCl}_{2}$; pH 6 (Trombitas and Pollack, 1995). Samples were embedded in Araldite 506. Ultrathin sections $(\sim 20 \mathrm{~nm})$ were cut with an LKB Ultrotome III. Sections were stained with potassium permanganate, and lead citrate, and observed and photographed with a Philips 420 electron microscope.

\section{RESULTS}

\section{MOUNTING TECHNIQUES FOR DROSOPHILA FLIGHT MUSCLE}

The length of the indirect flight muscle fibers of $D$. melanogaster is only $\sim 1 \mathrm{~mm}$, and it is very difficult to mount both ends with metal foil clips, as would often be done with longer specimens, while keeping the myofibrils straight (this is required to yield good diffraction patterns). 2-D diffraction patterns from Drosophila flight muscles have been recorded from whole live insects by using two configurations.

One is to attach a hollow tube on top of the thorax and irradiate X-rays through it (Irving and Maughan, 2000; Dickinson et al., 2005). By doing so one can obtain diffraction patterns from the dorsal longitudinal muscle (DLM) alone, one of the two antagonistic indirect flight muscles (Figure 1A). The other is to glue the whole insect, and irradiate X-rays from the side (Figure 1B; Iwamoto et al., 2007), similar to the configuration used for recording diffraction patterns from live bumblebees (Iwamoto and Yagi, 2013). In this configuration, one can simultaneously record diffraction patterns from both of the two antagonistic flight muscles (DLM and dorsoventral muscle, DVM) as well as the jump muscle. In Iwamoto et al. (2007) this configuration was used also for recording diffraction patterns from pupae to study the time course of development of flight muscle architecture.

The drawbacks of such in vivo X-ray recording are that (1) there are unwanted materials in the beampath (such as legs, leg muscles, nerves, digestive tracts, etc.) that could compromise the quality of diffraction patterns, and that (2) the solution environment of contractile proteins cannot be changed at will. For these reasons it is desirable to establish techniques to record diffraction patterns from demembranated (glycerinated) flight muscle specimens from Drosophila.

Here we tested several techniques to record diffraction patterns from glycerinated specimens, besides the conventional clamping technique. The first one is to use a stainless-steel plate with round holes. The glycerinated thorax was placed in the hole and glued by 

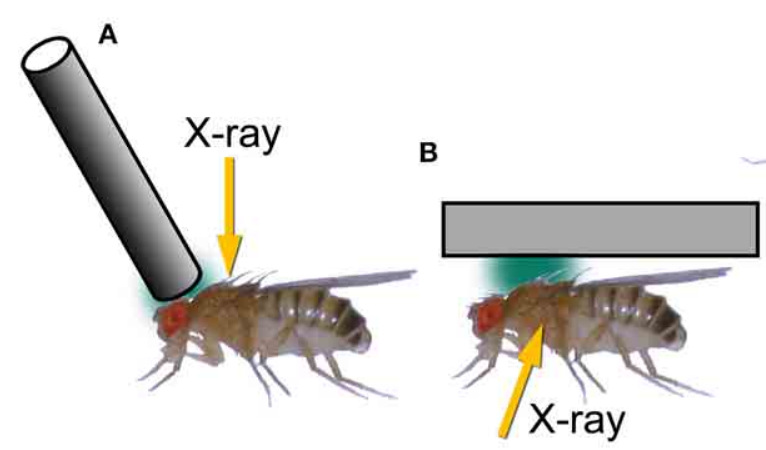

D

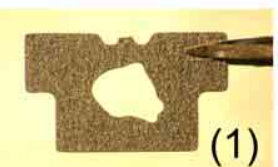

(1)
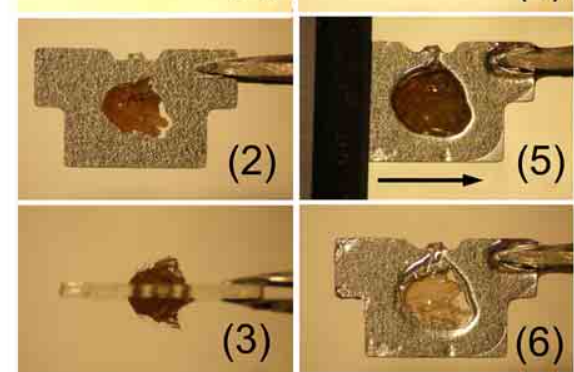

(4)

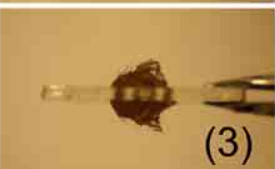

(3)
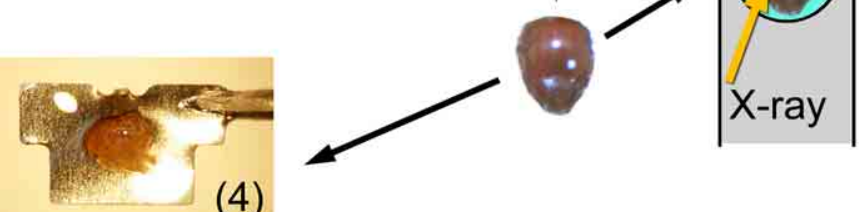

E

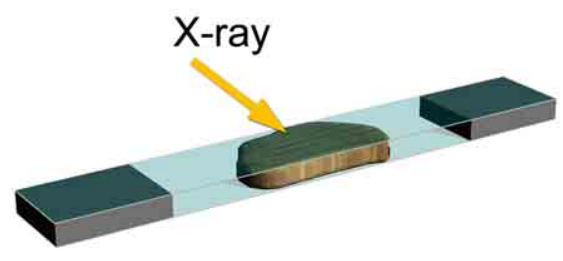

FIGURE 1 | Mounting techniques for Drosophila indirect flight muscle. (A,B) Methods to record X-ray diffraction patterns from live flies; (C-E) methods to record patterns from glycerinated muscle preparations. (A), attaching a metal wire on top of the head and at the anterior end of the thorax and the fly is irradiated with X-rays from the top. This is the method adopted by Irving and Maughan (2000), Dickinson et al. (2005). (B), a fly, glued to a substrate, is irradiated from the side to record patterns from both of the two antagonistic flight muscles (DLM and DVM). This is the configuration used by Iwamoto et al. (2007). (C), gluing an isolated thorax to a stainless-steel plate with round holes. After gluing, unwanted tissues, such as leg muscles and digestive tract, are removed. (D), procedure to glue an isolated thorax to a stainless-steel plate with a precision-etched hole and to remove excess parts of the thorax. (1), a plate before mounting; (2) an isolated thorax fitted to the hole; (3) top view; (4) thorax glued to the plate, (5) an ultrasonic-vibrated microtome blade slides along the surface of the plate; (6) finished sample with exposed DLM. (E), sandwiching an isolated flight muscle between two thin plastic films $(1.5 \mu \mathrm{m}$-thick polyester, Chemplex Industries, Palm City, USA). Incident $\mathrm{X}$-ray beams make a small angle with respect to the film plane. using a cyanoacrilic resin (Figure 1C). After gluing, the legs and other unwanted materials were removed. By irradiating X-rays in the middle of the hole, one can obtain diffraction patterns from DLM alone. The DLM fibers were irradiated from the top of the thorax.

The second technique tested was to fabricate stainless-steel plates (thickness, $0.2 \mathrm{~mm}$ ) by photo-etching, each with a hole shaped to fit the contour of the thorax. In this method, the orientation of DLM fibers can be precisely fixed with respect to the stainless-steel plate. The thorax was glued so that equal amounts of volume of the thorax stick out of both sides of the plate. After this, the parts of the thorax sticking out of the plate were cut off by using an ultrasonic vibration cutter with microtome blades. This leaves DLM fibers within the thickness of the plate. X-rays were irradiated from the side (Figure 1D).

The third technique was to isolate the assembly of 6 indirect flight muscle fibers (both DLM and DVM have 6 flight muscle fibers on each side), and sandwich them with two very thin plastic films (Figure 1E). The plastic films had been glued to two pieces of thin plastic plate at both ends, leaving a small gap between them so that the fibers were not crushed. X-ray beams were irradiated so that the beam axis made a small angle with respect to the film plane. By doing so one should be able to eliminate the parasitic scattering resulting from the total reflection from the film surface. However, the fact was that some parasitic scattering was recorded, probably because the film surface was somewhat wavy.

\section{DIFFRACTION PATTERNS RECORDED FROM WILD-TYPE DROSOPHILA FLIGHT MUSCLE}

Figure 2 shows some of the diffraction pattern recorded from the indirect flight muscles from wild type and mutant strains of D. melanogaster. For wild-type flies, the second technique (using photo-etched stainless steel plates) has so far given the best myofiber orientations (Figure 2A). This diffraction pattern was recorded in rigor (in the absence of ATP), and this sample seems to contain a small remnant of DVM as the pattern contains its equatorial reflections. Although there was some problem in data processing, good myofiber orientation was also obtained 

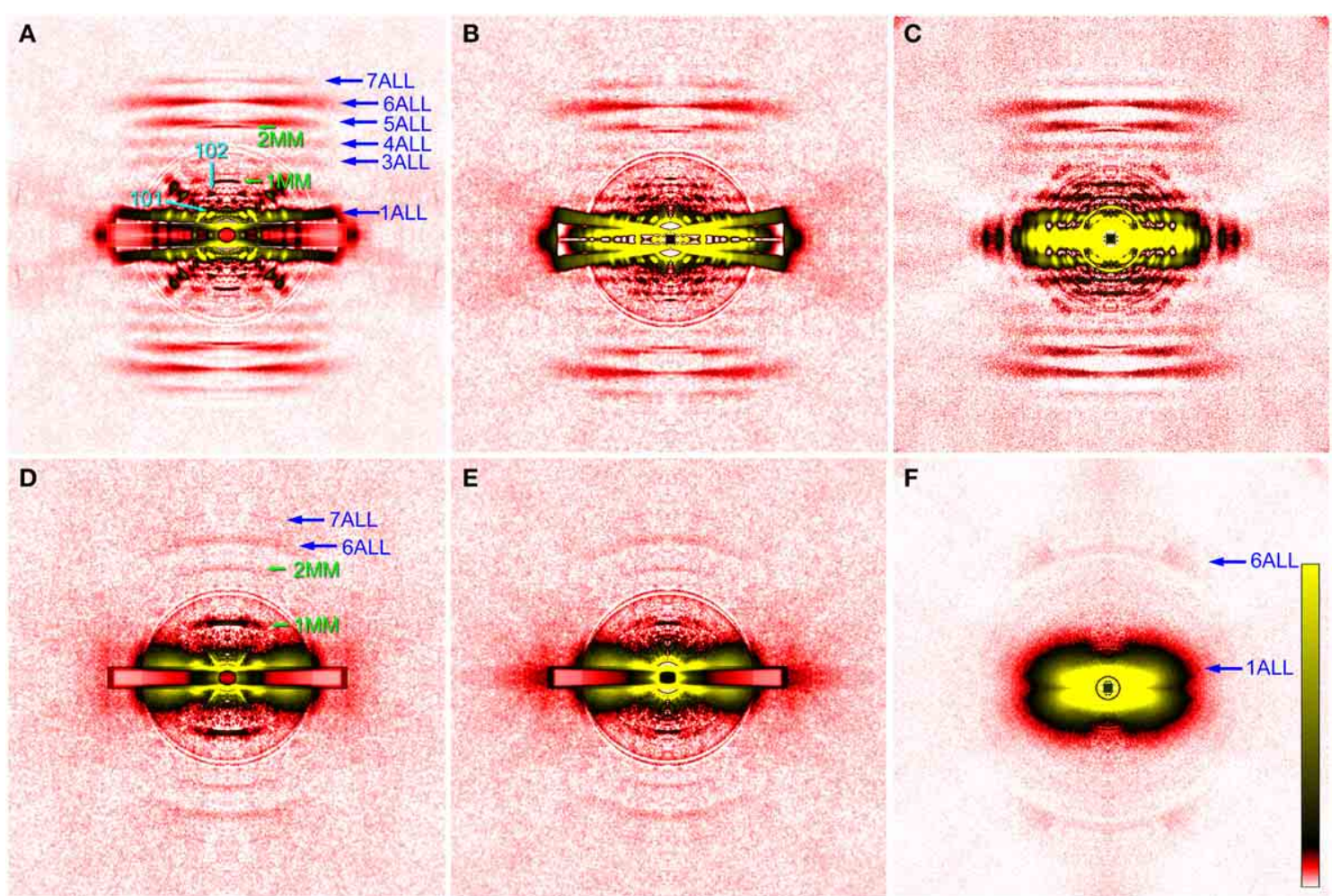

FIGURE 2 | X-ray diffraction patterns from the glycerinated flight muscle (DLM) of wild-type and mutant Drosophila. (A-C), wild type; (D,E), headless myosin mutant ( $\mathrm{MhC}^{10}$-Y97); (F) mutant with no myosin heavy chain $\left(M h c^{10}\right.$-background line). Patterns in (A-D) were recorded from single flies, and the pattern in $(\mathbf{F})$ is the sum of data from 9 flies. The mounting techniques used are: (A), 2nd (etched plate); (B,D), 3rd (sandwiching with plastic films); (C,F), 1st (round holes); (D), conventional clamping. The four quadrants of the pattern were folded and the background scattering was subtracted as described by Iwamoto et al. $(2003,2010)$. The circles around the center are due to the correction of absorption by round aluminum masks to attenuate the intense central parts of the pattern. In $(\mathbf{A}, \mathbf{B}, \mathbf{D}, \mathbf{E})$ a rectangular copper mask was also applied to the equator in which the scattering intensity was strongest. The oblique anomalous reflections in (A) come from the remnant of DVM. They are observed in four quadrants because the image has been averaged for the four quadrants. The aberration around the equator in $(\mathbf{B})$ is generated in the process of background subtraction because of the mismatch of fiber and copper mask orientations. $A L L$, actin layer line reflection; $M M$, myosin meridional reflection. The numbers indicate the order or Miller indices of reflections. Note that only actin-based reflections are observed in (F). with the third technique, i.e., to sandwich the fibers with plastic films (Figure 2B). The fiber orientation was worse with the first technique (Figure 2C), and the conventional (most laborintensive) technique of clamping both ends of isolated fibers gave totally unsatisfactory results (data not shown). The recorded diffraction pattern is fundamentally similar to that from flight muscle fibers of different insect species (e.g., Tregear et al., 1998; Bekyarova et al., 2008). The lower-angle layer line reflections are finely lattice-sampled, and unlike in relaxed or activated fibers, the 4th-7th actin layer lines (they have continuous intensities along the layer) are clearly visible, indicating that myosin heads are stereospecifically bound to actin following its periodicity.

As for the innermost reflection spots on the 1st and 2nd layer lines, i.e., the 101 and 102 reflections (cyan arrows in Figure 2), the 101 is much more intense than 102, in agreement with that for giant waterbug, Lethocerus (Tregear et al., 1998). These reflections are considered to arise from the helical arrangement of troponin complexes around a thick filament, and are known to change their intensities upon stretch activation. Before stretch activation, the 101 is stronger than 102, and vice versa after stretch activation (Tregear et al., 1998; Dickinson et al., 2005; Bekyarova et al., 2008; Iwamoto et al., 2010; Perz-Edwards et al., 2011). This is because a stretch induces stereospecific attachment of myosin heads to the actin target zone located midway between the two neighboring troponin complexes on a thin filament, and these heads negatively interfere with the basic $38.7-\mathrm{nm}$ periodicity of troponin complexes. In rigor, the 101 is intensified again, because excessive myosin binding to the actin target zones restores the 38.7-nm periodicity (Tregear et al., 1998). Together with the reciprocal behavior of these reflections in live flies (Dickinson et al., 2005), the present observations suggest that the numbers of attached myosin heads are similar in Drosophila and Lethocerus flight muscles, either in active or in rigor states.

The diffraction pattern from the wild-type indirect flight muscle also shows clear myosin meridional reflections based on the 14.5-nm repeat (green arrows in Figure 2). The intensity of the 1st meridional reflection is spread along the equator, in contrast to that of other insects which has a sharper peak right on the meridian (Bekyarova et al., 2008; Iwamoto, 2009). Although the peak splitting is not clear, the present observation agrees with the idea 
that the thick filaments form a super lattice with a larger lattice constant in Drosophila (Squire et al., 2006).

\section{DIFFRACTION PATTERNS RECORDED FROM THE HEADLESS MYOSIN MUTANT}

The indirect flight muscles of flies with myosin mutations, either myosin-null or headless, have structural defects (Cripps et al., 1999) and are more fragile than those from wild-type flies. Therefore, slicing with vibrating blades often resulted in unsatisfactory processing. The diffraction patterns from the headless mutant as shown in Figure 2 were recorded from either clamped (Figure 2D) or sandwiched (the 3rd procedure, Figure 2E) flight muscle fibers. The two techniques gave equivalent results.

Compared with the wild type, the pattern from the $M h c^{10}$ $Y 97$ (headless myosin) flight muscle is much more featureless, indicating that the structure is disorganized. Lattice sampling of layer-line reflections are not recognized. However, the 6th and 7 th actin layer line reflections are clearly visible. The arcing of these reflections indicates that actin filaments are not well oriented in this mutant. Although it is not clear from the pattern, equatorial reflections are present as their intensity profiles are shown in Figure 3 (red). This indicates that the hexagonal lattice arrangement of myofilaments is maintained in this mutant.

Most notable in the pattern from the $M h c^{10}-Y 97$ indirect flight muscle is the very strong 1 st myosin meridional reflection at $14.5 \mathrm{~nm}^{-1}$. The $2 \mathrm{nd}$ myosin meridional reflection at $7.2 \mathrm{~nm}^{-1}$ is also clearly visible. In the diffraction pattern from the $M h c^{10}$ background line (missing the entire myosin heavy chain), no meridional reflection was observed at $14.5 \mathrm{~nm}^{-1}$ (Figure 2F). This pattern was taken by using the first procedure. In this pattern, the strong 1st actin layer line and the weaker 6th actin layer line are observed. The latter is strongly arced, indicating that

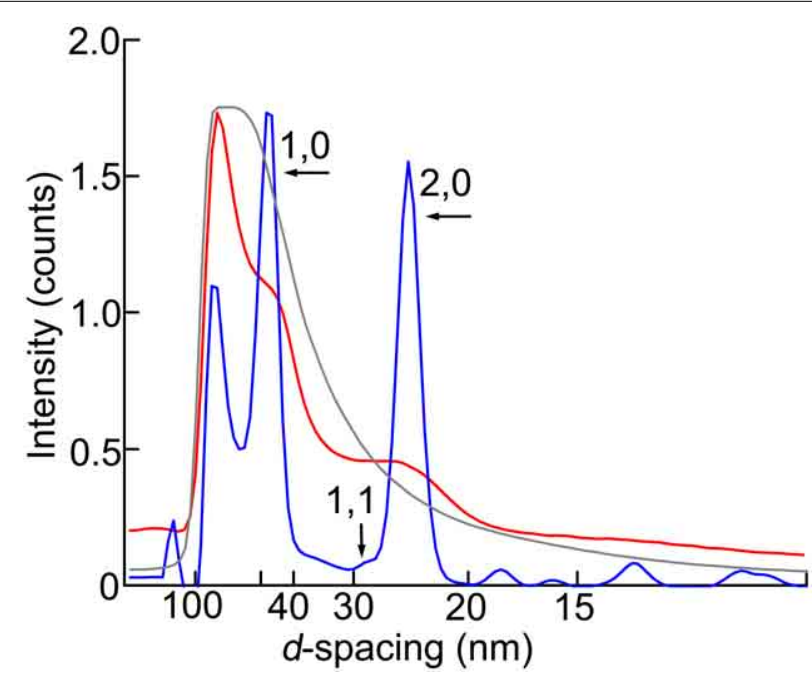

FIGURE 3 | Intensity profiles of the equatorial reflection. Blue, wild type; red, headless myosin mutant (Mhc ${ }^{10}-$ Y97); gray, mutant with no myosin heavy chain ( $M h c^{10}$-background line). The maximal intensity counts (digitized bits of analog-to-digital converter output) are $2.0 \times 10^{6}$ for wild type, $2.0 \times 10^{5}$ for $\mathrm{Mhc}^{10}-$ Y97, and $2.8 \times 10^{7}$ for the $M h c^{10}$-background line. the actin filament orientation is less organized in the absence of myosin filaments.

To make quantitative comparisons, the integrated intensity of the 1st myosin meridional reflection was normalized to that of the 6th actin layer line reflection, which is often used as an internal standard to compare intensities of various reflections (e.g., Iwamoto, 2009). Its intensity was $76 \pm 12 \%$ (mean \pm S.D., $n=5$ ) of that of the 6 th actin layer line. Although the patterns were recorded in the absence of ATP, the $M h c^{10}-Y 97$ myosin cannot form rigor linkage to the thin filaments, and therefore the values should be compared with the values from relaxed wild-type flight muscle fibers. In Drosophila it was difficult to obtain an ideally relaxed pattern, probably because of the insufficient supply of ATP due to myosin's high ATPase activity (Swank et al., 2006). Instead, we used flight muscle fibers from a bigger dipteran, Tabanus trigonus (a horse fly), to obtain relaxed patterns (Figure 4). We recorded patterns from two sets of flight muscle fibers and obtained the intensity ratios of 59 and $64 \%$, which are lower than the values for $M h c^{10}-Y 97$. Because of the disorder of actin filament orientations in $M h c^{10}-Y 97$, some of scattering intensities may have been lost to the background scattering, and the intensity of the 6th actin layer line may be underestimated. However, it still holds true that the 1st myosin meridional reflection is unexpectedly strong if its major source is the motor domain of the myosin molecule (see Discussion).

\section{ELECTRON MICROSCOPY}

The rationale for using the 6th actin layer line reflection as an internal standard is that the $M h c^{10}-Y 97$ mutant fly retains the same thick-to-thin filament number ratio as in the wild type, in which it is $1: 3$. To verify this, the cross section of myofibrils of

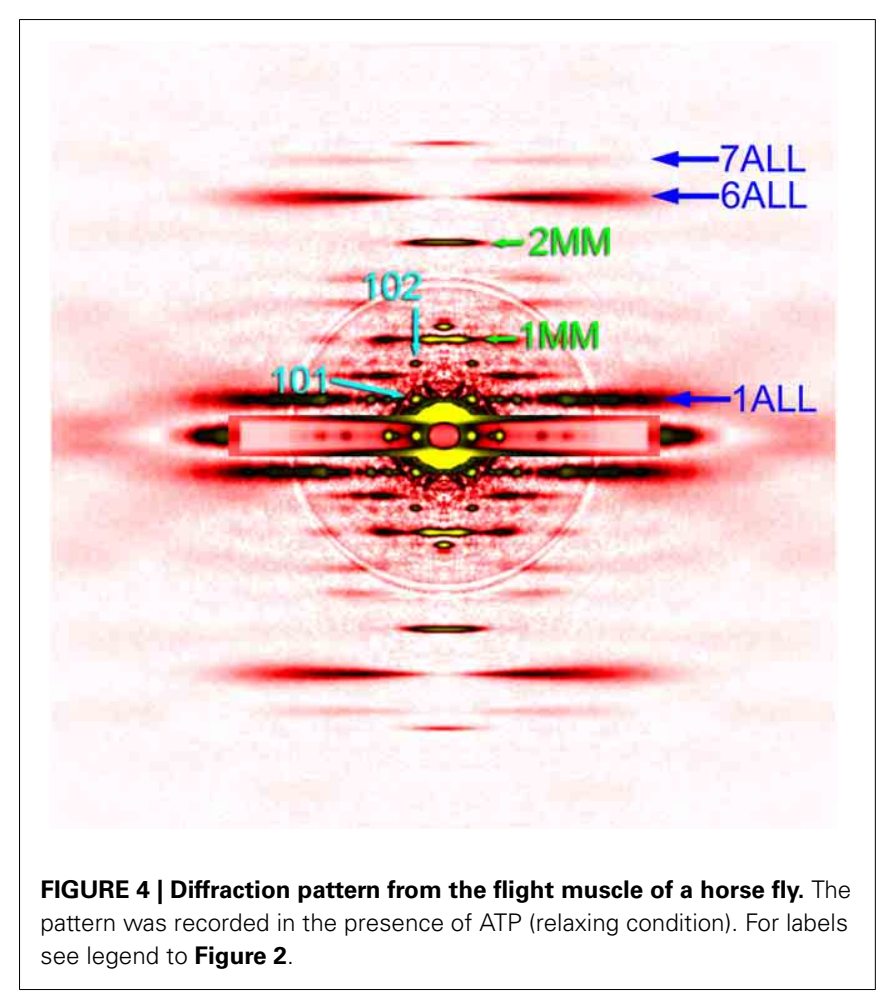




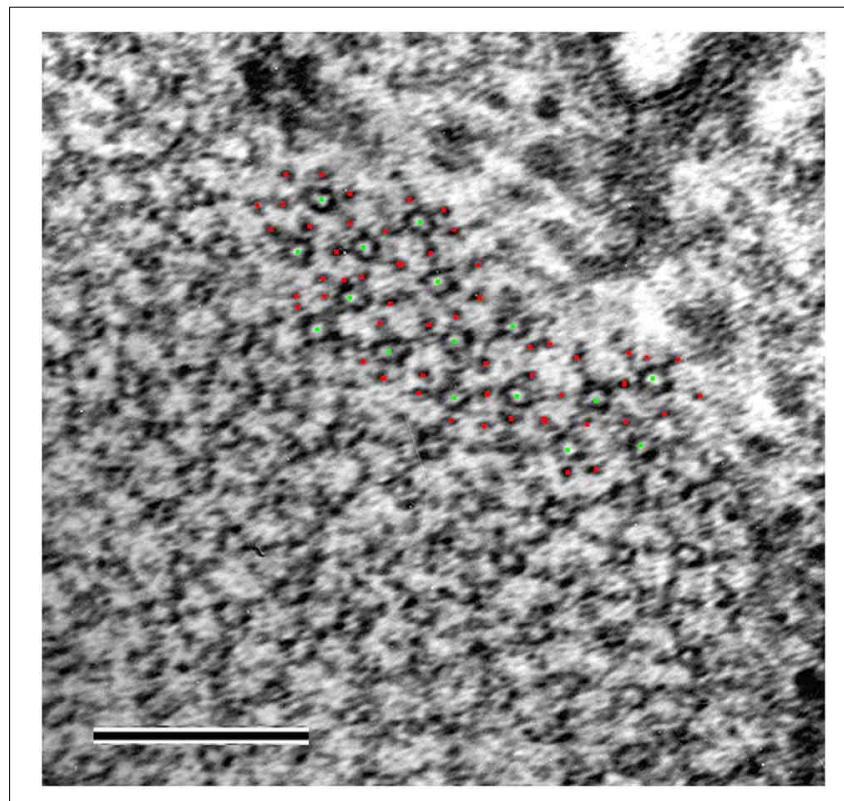

FIGURE 5 | Electron micrograph of a myofibril in transverse orientation from the headless myosin mutant. The green and red dots represent the positions of the thick and thin filaments as recognized by eye. Scale bar, $200 \mathrm{~nm}$.

the $M h c^{10}-Y 97$ flight muscle was observed by electron microscopy (Figure 5). Because of the waviness of the myofibrils, clear cross sections of both thick and thin filaments were observed in limited areas. In such an area, the numbers of the recognized thick and thin filaments were 16 (green dots in Figure 5) and 51 (red dots), respectively. This is close to the 1:3 ratio. It therefore appears that the thick-to-thin filament ratio is preserved in the $M h c^{10}-Y 97$ mutant.

The X-ray observation of the strong myosin meridional reflection and the preserved thick-to-thin filament ratio strongly suggest the presence of periodic structures with strong contrast with a $14.5 \mathrm{~nm}$ spacing even in the absence of most of the myosin motor domain. We therefore tested whether such periodic structures were visible in the longitudinal sections of myofibrils from the $M h c^{10}-Y 97$ indirect flight muscle (Figure 6).

Figure 6A shows one of the longitudinal sections of the mutant myofibril. The picture in Figure $\mathbf{6 B}$ is a running-average of the micrograph in Figure 6A, calculated by superposing itself multiple times after translating by a fixed distance. By doing so, features are enhanced if its periodicity coincides with the distance of translation. The summed picture in Figures 6B,C clearly shows an enhanced feature with a periodicity near $14.5 \mathrm{~nm}$. An alternative way of demonstrating the presence of periodic structures is to calculate a power spectrum of the micrograph, by applying Fourier transformation along the filament axis. The power spectrum calculated from the micrograph in Figure 6A clearly shows a peak at $\sim 14.5 \mathrm{~nm}$ along with a strong peak at $\sim 38.7 \mathrm{~nm}$, which arises from troponin and tropomyosin on the thin filament (Figure 6D). These results indicate that there are structures with a $14.5-\mathrm{nm}$ structure that are visible by both X-ray and electron microscopy. If there were intact myosin heads, they would

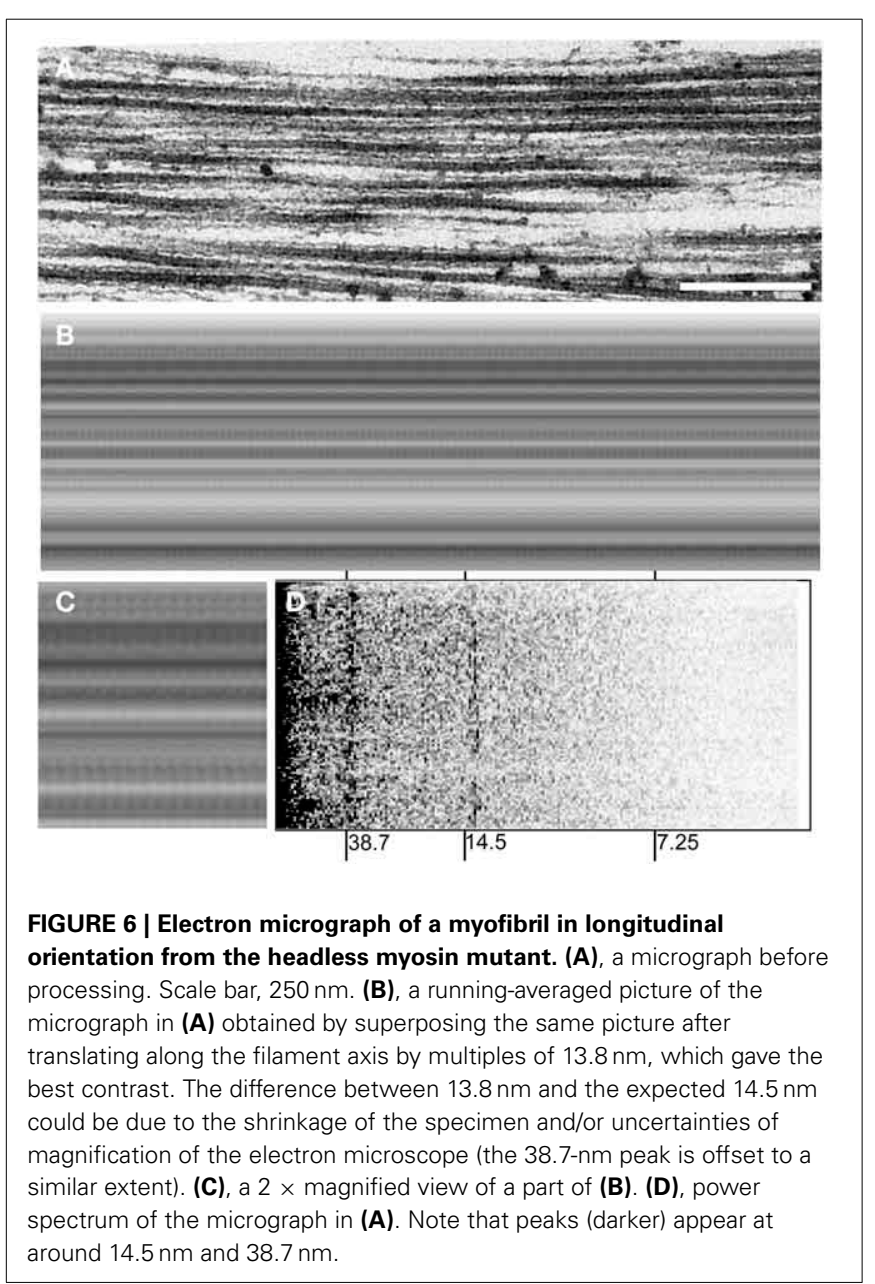

bind to actin target zones (these have a 38.7-nm periodicity) and therefore they would contribute to the $38.7-\mathrm{nm}$ peak, rather than 14.5-nm.

\section{DISCUSSION}

In this paper several methods to record X-ray diffraction patterns from glycerinated flight muscle fibers of Drosophila are described, and these methods were applied to the wild-type and the $M h c^{10}-Y 97$ (headless myosin) mutant flies. We have shown that, by the use of intense $\mathrm{X}$-rays generated by the 3rd-generation synchrotron radiation facilities, one can record high-quality 2dimensional diffraction patterns from glycerinated flight muscle fibers. The most important finding is that intense myosin meridional reflections are recorded from the $M h c^{10}-Y 97$ mutant flies in which most of the myosin motor domain is missing.

\section{THE BEST MOUNTING METHOD FOR DROSOPHILA FLIGHT MUSCLE}

For wild-type flies, the best-quality patterns were obtained by the second method, i.e., to glue the thorax to a photo-etched stainless steel plate and to remove excess parts by an ultrasonic-vibrated blade. The merit of this method is that only the DLM remains in the hole with its in-situ length, if the thorax has been glued in a proper position (see Figure 1D3) and the blade is moved along the surface of the plate without a gap (the plate is $0.2-\mathrm{mm}$ thick). 
In contrast to the two other methods, both sides of the flight muscle fibers are exposed to the surrounding medium, so that this method is most suitable for solution exchange experiments.

This method was successfully applied to wild-type flies, probably because of the physical strength of their flight muscle fibers. Although this method was not successfully applied to the more fragile mutant muscles, it is still a promising mounting method for solution exchange for mutant muscles. Improvements of this method, such as automated controlled blade movements (the blades were moved manually in this work) and/or increasing the physical strength of fibers by soaking in glycerol and slicing at low temperatures, may allow us to apply this method also to mutant flight muscles. The throughput of X-ray recording may be increased by creating stainless-steel strips with multiple photo-etched holes (see Figure 1C) instead of just one as in this study.

\section{THE ORIGIN OF THE REFLECTIONS BASED ON A 14.5-nm-SPACING IN THE MUTANT FLY}

The most important finding in this study is the strong myosinbased meridional reflections (at 14.5 and $7.2 \mathrm{~nm}^{-1}$ ) observed in the $M h c^{10}-Y 97$ mutant, in which most of the motor domain is missing except for a part of the lever arm and the regulatory light chain. The $14.5 \mathrm{~nm}$-spaced features are also evident in electron micrographs (Figure 6). The strong myosin meridional reflections are unexpected because it is generally believed that the major source of this reflection is the motor domain of the myosin molecule, while the rod domain may contribute to the 2nd reflection (Huxley et al., 1994; Lombardi et al., 2004).

As mentioned briefly in Results, the intensity of the myosin meridional reflection is compared with that of the 6 th actin layer line reflection, which might be underestimated because of the disorder. Indeed, the 6th actin layer line seems to be weaker in the mutant, but it is partly explained by the smaller myofibrillar content in the mutant flight muscle cell as shown by Cripps et al. (1999), Figure 6C. On the other hand, the 1st myosin meridional reflection in the mutant pattern is at least as strong as that in the wild type in rigor, despite that its intensity may also be underestimated for the same reason for the 6th actin layer line. Therefore, it still holds true that the 1 st myosin meridional reflection is unexpectedly strong.

The question remains whether there are other proteins on the thick filament with a $14.5-\mathrm{nm}$ periodicity. In vertebrate striated muscles, it is known that myosin binding protein C (MyBP-C) has a 42.9-nm periodicity (Rome et al., 1973), and its 3rd-order reflection is expected to overlap with the 1st myosin meridional reflection. MyBP-C is absent from insect flight muscle, but instead, a myosin binding protein flightin has been reported (Vigoreaux et al., 1993). However, the molecular weight of flightin is only $20 \mathrm{kDa}$ and it is unlikely that it contributes significantly to the intensity of the meridional reflection at $14.5 \mathrm{~nm}^{-1}$. Another possibility is the remaining myosin lever arm region containing the regulatory light chain (RLC). The RLC has an N-terminal extension that has been suggested to serve in parallel to the motor domain as a link to the thin filament (Moore et al., 2000; Miller et al., 2011). This is similar to the observation that the essential light chain extension of vertebrates appears to bind to actin within thin filaments (Miller et al., 2005; Lowey et al., 2007). If this extension does not preferentially bind to the actin target zone but simply splays out radially, it could strengthen the 1st myosin meridional reflection to some extent. However, the extension is only 50 residues long and again it is unlikely to contribute significantly to the intensity. In fact, removal of the Drosophila RLC extension does not diminish the intensity of the 1st myosin meridional reflection (Farman et al., 2009). Insect muscles are known to contain paramyosin, and it may also contribute to some extent to the 1st myosin meridional reflection. Again, however, the paramyosin content in Drosophila flight muscle is small (myosin:paramyosin $=34: 1$; Vinós et al., 1991), and its structural resemblance to the rod portion of myosin makes it unlikely that paramyosin contributes significantly to the 1st myosin meridional reflection.

From the considerations above, we are currently forced to consider that the strong $14.5 \mathrm{~nm}$-spaced feature arise from the truncated myosin heavy chain and the regulatory light chain. It is generally believed that the rod portions of two myosin heavy chains form a coiled-coil structure to form a dimer, and their periodic clusters of charged residues allow the dimers to assemble into thick filaments with a stagger of $14.5 \mathrm{~nm}$ (McLachlan and Karn, 1982). There could be a different extent of deviation from the ideal coiled-coil structure between the charged and noncharged regions, and it could contribute to the intensity of the 1st myosin meridional reflection. The present results could indicate that the contribution of such a structural deviation to the 1st myosin meridional reflection is substantial. Conclusive explanations cannot be given from the present results alone, and further experiments are needed to address this issue. These experiments include the creation of a double mutant with $\left(M h c^{10}-Y 97\right)$ and a knockout of either flightin or RLC or both. It is also important to refine the present method for X-ray diffraction recording to ensure better myofibrillar orientations; it would reduce the ambiguity arising from the poorer filament orientation of actin as compared with myosin.

\section{CONCLUSION}

Here we have demonstrated that the flight muscle of $D$. melanogaster can serve as a model to study the causes and effects of myosin myopathies, and their structural consequences can be monitored by X-ray diffraction. This should be applicable to studying models of myosin-based congenital myopathies (Wang et al., 2012). In the particular case of the myosin headless mutation $\left(M h c^{10}-Y 97\right)$, an unexpected observation of the strong feature with a $14.5-\mathrm{nm}$ periodicity prompts us to reinvestigate the origin of the 1st myosin meridional reflection, one of the best-studied X-ray reflections of muscle.

\section{ACKNOWLEDGMENTS}

We thank Dr. T. Hikima (RIKEN) for his support at the BL45XU beamline of SPring-8, Dr. Gerald Pollack (University of Washington) and Dr. Karen Hsu (San Diego State University) for helpful discussions and Dr. Henk Granzier (University of Arizona) for access to electron microscopy facilities at Washington State University. The experiments were performed under approval of the SPring-8 Proposal Review Committee 
(Proposal Nos. 2009B1274, 2010A1230, 2010B1263, 2011A1242). Supported by Grant-in-Aid for Scientific Research, The Ministry of Education, Culture, Sports, Science and Technology, Japan, No. 23612009 and 26440185 and by United States National Institutes of Health R01 Grants AR43396, GM32443 and HL062881.

\section{REFERENCES}

Bekyarova, T. I., Reedy, M. C., Baumann, B. A. J., Tregear, R. T., Ward, A., Krzic, U., et al. (2008). Reverse actin sliding triggers strong myosin binding that moves tropomyosin. Proc. Natl. Acad. Sci. U.S.A. 105, 10372-10377. doi: 10.1073/pnas.0709877105

Bernstein, S. I., O’Donnell, P. T., and Cripps, R. M. (1993). Molecular genetic analysis of muscle development, structure, and function in Drosophila. Int. Rev. Cytol. 143, 63-152. doi: 10.1016/S0074-7696(08)61874-4

Collier, V. L., Kronert, W. A., O’Donnell, P. T., Edwards, K. A., and Bernstein, S. I. (1990). Alternative myosin hinge regions are utilized in a tissue-specific fashion that correlates with muscle contraction speed. Genes Dev. 4, 885-895. doi: 10.1101/gad.4.6.885

Corpeno, R., Dworkin, B., Cacciani, N., Salah, H., Bergman, H.-M., Ravara, B., et al. (2014). Time-course analysis of mechanical ventilation-induced diaphragm contractile muscle dysfunction in the rat. J. Physiol. 592, 3859-3880. doi: 10.1113/jphysiol.2014.277962

Cripps, R. M., Suggs, J. A., and Bernstein, S. I. (1999). Assembly of thick filaments and myofibrils occurs in the absence of the myosin head. EMBO J. 18, 1793-1804. doi: 10.1093/emboj/18.7.1793

Dickinson, M., Farman, G., Frye, M., Bekyarova, T., Gore, D., Maughan, D., et al. (2005). Molecular dynamics of cyclically contracting insect flight muscle in vivo. Nature 433, 330-333. doi: 10.1038/nature03230

Farman, G. P., Miller, M. S., Reedy, M. C., Soto-Adames, F. N., Vigoreaux, J. O., Maughan, D. W., et al. (2009). Phosphorylation and the N-terminal extension of the regulatory light chain help orient and align the myosin heads in Drosophila flight muscle. J. Struct. Biol. 168, 240-249. doi: 10.1016/j.jsb.2009.07.020

Fujisawa, T., Inoue, K., Oka, T., Iwamoto, H., Uruga, T., Kumasaka, T., et al. (2000). Small-angle X-ray scattering station at the SPring-8 RIKEN beamline. J. Appl. Cryst. 33, 797-800. doi: 10.1107/S002188980000131X

Huxley, H. E., and Brown, W. (1967). The low-angle X-ray diagram of vertebrate striated muscle and its behaviour during contraction and rigor. J. Mol. Biol. 30, 383-434. doi: 10.1016/S0022-2836(67)80046-9

Huxley, H. E., Stewart, A., Sosa, H., and Irving, T. (1994). X-ray diffraction measurements of the extensibility of actin and myosin filaments in contracting muscle. Biophys. J. 67, 2411-2421. doi: 10.1016/S0006-3495(94)80728-3

Irving, T. C., and Maughan, D. W. (2000). In vivo X-ray diffraction of indirect flight muscle from Drosophila melanogaster. Biophys. J. 78, 2511-2515. doi: 10.1016/S0006-3495(00)76796-8

Iwamoto, H. (2000). Influence of ionic strength on the actomyosin reaction steps in contracting skeletal muscle fibers. Biophys. J. 78, 3138-3149. doi: 10.1016/S0006-3495(00)76850-0

Iwamoto, H. (2009). Evidence for unique structural change of the thin filaments upon calcium-activation of insect flight muscle. J. Mol. Biol. 390, 99-111. doi: 10.1016/j.jmb.2009.05.002

Iwamoto, H., Inoue, K., Matsuo, T., and Yagi, N. (2007). Flight muscle myofibrillogenesis in the pupal stage of Drosophila as examined by X-ray microdiffraction and conventional diffraction. Proc. Biol. Sci. 274, 2297-2305. doi: 10.1098/rspb.2007.0607

Iwamoto, H., Inoue, K., and Yagi, N. (2010). Fast X-ray recordings reveal dynamic action of contractile and regulatory proteins in stretch-activated insect flight muscle. Biophys. J. 99, 184-192. doi: 10.1016/j.bpj.2010.04.009

Iwamoto, H., Oiwa, K., Suzuki, T., and Fujisawa, T. (2001). X-ray diffraction evidence for the lack of stereospecific protein interactions in highly activated actomyosin complex. J. Mol. Biol. 305, 863-874. doi: 10.1006/jmbi.2000.4334

Iwamoto, H., Wakayama, J., Fujisawa, T., and Yagi, N. (2003). Static and dynamic X-ray diffraction recordings from living mammalian and amphibian skeletal muscles. Biophys. J. 85, 2492-2506. doi: 10.1016/S0006-3495(03) 74672-4

Iwamoto, H., and Yagi, N. (2013). The molecular trigger for high-speed wing beats in a bee. Science 341, 1243-1246. doi: 10.1126/science.1237266

Lindqvist, J., Iwamoto, H., Blanco, G., and Ochala, J. (2013). The fraction of strongly bound cross-bridges is increased in mice that carry the myopathy-linked myosin heavy chain mutation MYH4L342Q. Disease Models Mech. 6, 834-840. doi: 10.1242/dmm.011155

Lindqvist, J., Pénisson-Besnier, I., Iwamoto, H., Li, M., Yagi, N., and Ochala, J. (2012). A myopathy-related actin mutation increases contractile function. Acta Neuropathol. 123, 739-746. doi: 10.1007/s00401-012-0962-z

Lombardi, V., Piazzesi, G., Reconditi, M., Linari, M., Lucii, L., Stewart, A., et al. (2004). X-ray diffraction studies of the contractile mechanism in single muscle fibres. Phil. Trans. R. Soc. B 359, 1883-1893. doi: 10.1098/rstb. 2004.1557

Lowey, S., Saraswat, L. D., Liu, H., Volkmann, N., and Hanein, D. (2007). Evidence for an interaction between the SH3 domain and the N-terminal extension of the essential light chain in class II myosins. J. Mol. Biol. 371, 902-913. doi: 10.1016/j.jmb.2007.05.080

McLachlan, A. D., and Karn, J. (1982). Periodic charge distributions in the myosin rod amino acid sequence match cross-bridge spacings in muscle. Nature 299, 226-231. doi: 10.1038/299226a0

Miller, M. S., Farman, G. P., Braddock, J. M., Soto-Adames, F. N., Irving, T. C., Vigoreaux, J. O., et al. (2011). Regulatory light chain phosphorylation and N-terminal extension increase cross-bridge binding and power output in Drosophila at in vivo myofilament lattice spacing. Biophys. J. 100, 1737-1746. doi: 10.1016/j.bpj.2011.02.028

Miller, M. S., Palmer, B. M., Ruch, S., Martin, L. A., Farman, G. P., Wang, Y. et al. (2005). The essential light chain N-terminal extension alters force and fiber kinetics in mouse cardiac muscle. J. Biol. Chem. 280, 34427-34434. doi: 10.1074/jbc.M508430200

Moore, J. R., Dickinson, M. H., Vigoreaux, J. O., and Maughan, D. W. (2000). The effect of removing the N-terminal extension of the Drosophila myosin regulatory light chain upon flight ability and the contractile dynamics of indirect flight muscle. Biophys. J. 78, 1431-1440. doi: 10.1016/S0006-3495(00)76696-3

Ochala, J., Gokhin, D. S., Iwamoto, H., and Fowler, V. M. (2014). Pointed-end capping by tropomodulin modulates actomyosin crossbridge formation in skeletal muscle fibers. FASEB J. 28, 408-415. doi: 10.1096/fj.13-239640

Ochala, J., and Iwamoto, H. (2013). Myofilament lattice structure in presence of a skeletal myopathy-related tropomyosin mutation. J. Muscle Res. Cell Motil. 34, 171-175. doi: 10.1007/s10974-013-9345-x

Ochala, J., Iwamoto, H., Larsson, L., and Yagi, N. (2010). A myopathylinked tropomyosin mutation severely alters thin filament conformational changes during activation. Proc. Natl. Acad. Sci. U.S.A. 107, 9807-9812. doi: 10.1073/pnas.1001733107

Ochala, J., Iwamoto, H., Ravenscroft, G., Laing, N. G., and Nowak, K. J. (2013). Skeletal and cardiac $\alpha$-actin isoforms differently modulate myosin cross-bridge formation and myofibre force production. Hum. Mol. Genet. 22, 4398-4404. doi: $10.1093 / \mathrm{hmg} / \mathrm{ddt} 289$

Ochala, J., Lehtokari, V. L., Iwamoto, H., Li, M., Feng, H.-Z., Jin, J.-P., et al. (2011). Disrupted myosin cross-bridge cycling kinetics triggers muscle weakness in nebulin-related myopathy. FASEB J. 25, 1903-1913. doi: 10.1096/fj.10176727

Oiwa, K., Kamimura, S., and Iwamoto, H. (2009). X-ray fiber diffraction studies on flagellar axonemes. Method Cell Biol. 91, 89-109. doi: 10.1016/S0091679X(08)91005-0

Peckham, M., Molloy, J. E., Sparrow, J. C., and White, D. C. (1990). Physiological properties of the dorsal longitudinal flight muscle and the tergal depressor of the trochanter muscle of Drosophila melanogaster. J. Muscle Res. Cell Motil. 11, 203-215. doi: 10.1007/BF01843574

Perz-Edwards, R. J., Irving, T. C., Baumann, B. A. J., Gore, D., Hutchinson, D. C., Krzic, U., et al. (2011). X-ray diffraction evidence for myosin-troponin connections and tropomyosin movement during stretch activation of insect flight muscle. Proc. Natl. Acad. Sci. U.S.A. 108, 120-125. doi: 10.1073/pnas.1014 599107

Rome, E., Offer, G., and Pepe, F. A. (1973). X-ray diffraction of muscle labelled with antibody to C-protein. Nat. New Biol. 244, 152-154. doi: 10.1038/newbio244152a0

Squire, J. M., Bekyarova, T., Farman, G., Gore, D., Rajkumar, G., Knupp, C., et al. (2006). The myosin filament superlattice in the flight muscles of flies: aband lattice optimisation for stretch-activation? J. Mol. Biol. 361, 823-838. doi: 10.1016/j.jmb.2006.06.072

Swank, D. M., Vishnudas, V. K., and Maughan, D. M. (2006). An exceptionally fast actomyosin reaction powers insect flight muscle. Proc. Natl. Acad. Sci. U.S.A. 103, 17543-17547. doi: 10.1073/pnas.0604972103 
Tregear, R. T., Edwards, R. J., Irving, T. C., Poole, K. J. V., Reedy, M. C., Schmitz, H., et al. (1998). X-ray diffraction indicates that active cross-bridges bind to actin target zones in insect flight muscle. Biophys. J. 74, 1439-1451. doi: 10.1016/S0006-3495(98)77856-7

Trombitas, K., and Pollack, G. H. (1995). Actin filaments in honeybeeflight muscle move collectively. Cell Motil. Cytoskeleton 32, 145-150. doi: $10.1002 / \mathrm{cm} .970320215$

Vigoreaux, J. O., Saide, J. D., Valgeirsdottir, K., and Pardue, M. L. (1993). Flightin, a novel myofibrillar protein of Drosophila stretch-activated muscles. J. Cell Biol. 121, 587-598. doi: 10.1083/jcb.121.3.587

Vinós, J., Domingo, A., Marco, R., and Cervera, M. (1991). Identification and characterization of Drosophila melanogaster paramyosin. J. Mol. Biol. 220, 687-700. doi: 10.1016/0022-2836(91)90110-R

Wang, Y., Melkani, G. C., Suggs, J. A., Melkani, A., Kronert, W. A., Cammarato, A., et al. (2012). Expression of the inclusion body myopathy 3 mutation in Drosophila depresses myosin function and stability and recapitulates muscle inclusions and weakness. Mol. Biol. Cell 23, 2057-2065. doi: 10.1091/mbc.E1202-0120
Conflict of Interest Statement: The authors declare that the research was conducted in the absence of any commercial or financial relationships that could be construed as a potential conflict of interest.

Received: 05 September 2014; accepted: 08 October 2014; published online: 29 October 2014.

Citation: Iwamoto H, Trombitás K, Yagi N, Suggs JA and Bernstein SI (2014) Xray diffraction from flight muscle with a headless myosin mutation: implications for interpreting reflection patterns. Front. Physiol. 5:416. doi: 10.3389/fphys.2014.00416 This article was submitted to Striated Muscle Physiology, a section of the journal Frontiers in Physiology.

Copyright (c) 2014 Iwamoto, Trombitás, Yagi, Suggs and Bernstein. This is an open-access article distributed under the terms of the Creative Commons Attribution License (CC BY). The use, distribution or reproduction in other forums is permitted, provided the original author(s) or licensor are credited and that the original publication in this journal is cited, in accordance with accepted academic practice. No use, distribution or reproduction is permitted which does not comply with these terms. 